\title{
On the issue of the installation of the purification degree dependence of polluted spent mineral oil on the hydrocyclone constructive and geometrical parameters
}

\author{
Andrey Glushchenko ${ }^{1}$, Denis Molochnikov ${ }^{1}$, Alexei Khokhlov ${ }^{1}$, Evgeniy Proshkin ${ }^{1}$, and Ilnar Gayaziev ,** $^{2}$ \\ ${ }^{1}$ Ulyanovsk State Agrarian University named after P.A. Stolypin, 432017 Ulyanovsk, Russia \\ ${ }^{2}$ Kazan State Agrarian University, 420015 Kazan, the Republic of Tatarstan, Russia
}

\begin{abstract}
The urgency of the issue is reasoned by the necessity to develop environmentally friendly technologies and engineering tools for cleaning emerged mineral oil from insoluble impurities in order to reuse them in the nodes and auto-tractor equipment systems. Hydrocyclone is one of the simplest and most effective means of cleaning waste oil. In order to determine the possibility of hydrocyclone usage in technological lines for purifying oils, it is necessary to establish the influence of its geometrical parameters on the purification quality of such high-viscosity liquid as emerged mineral oils. The purpose of the article is to study theoretically the cylindro-conical hydrocyclone geometrical parameters influence on the purification degree of emerged high-viscosity mineral oils from insoluble and wear debris. The leading approach to the study of this problem is the consideration of the behavior of a non-soluble particle in an oil stream under the influence of forces acting on it, which allows identifying the geometric and regime parameters of a cylindro-conical hydrocyclon. These parameters have the greatest influence on the oil purification degree. The article presents a theoretical justification for the geometrical parameters influence of a cylindrical-conical hydrocyclone on the emerged oil purification degree, a separation criterion, which allows not only determining the optimal size of a hydrocyclone, but also conducting a comparative assessment of hydrocyclones with different geometric parameters by oil purification efficiency from insolubles. Theoretical researches are aimed at determining the cylindro-conical hydrocyclone geometric parameters, depending on the pollution intensity of the emerged mineral oil and the desired degree of its purification from insolubles.
\end{abstract}

\section{Introduction}

The principle of oils and various technical fluids of purification and drying in force fields remains one of the most common. Various kinds of centrifuges and separators are widely used for this purpose. The principal disadvantage of all centrifuges and separators is the quality deterioration of oil purification at its lowering temperature, the devices complexity and low reliability $[1,2]$.

Recently, hydrocyclone units have been increasingly used for the various liquids purification, where solid particles are released from the purified oil stream under the influence of centrifugal forces. Hydrocyclone units are more reliable because they have no rotating parts, low cost, and easy maintenance. They can be installed in various places without perpendicular [3, 4]. Nowadays, many research centers are working on the hydrocyclone units' use for the various liquids purification.

A hydrocyclone (Fig. 1) is an apparatus consisting of a cylindrical part 1 , to which a conical part 7 adjoins the bottom with a wide base, and an intermediate drain chamber 3 with a branch pipe for discharging the top product. The diaphragm 6 is installed between the cylindrical part and the drain chamber, and replaceable nozzles 8 are fixed in the lower part of the cone.

Hydrocyclone for the purification of the emerged motor oil works as follows. Polluted oil under pressure enters through a device for admission 3 of cleaning oil tangentially into the cylindrical part of the body 1 and, acquiring a rotational motion, moves into the conical part of the body 1 . This causes significant centrifugal forces exceeding gravity.

The heavier fractions of the purified oil under centrifugal forces move from the hydrocyclone axis to the walls of its body 1 in a spiral trajectory downward. Then the heavy fractions are removed from the hydrocyclone through the drain pipe 7 . The lighter fractions move in the inner spiral flow, directed upwards, inside the diaphragm 2 . Then they enter to the internal part of the lid 5 and are removed through the device for draining cleaned oil 4 outside.

\footnotetext{
*Corresponding author: gazel.81@ mail.ru
} 


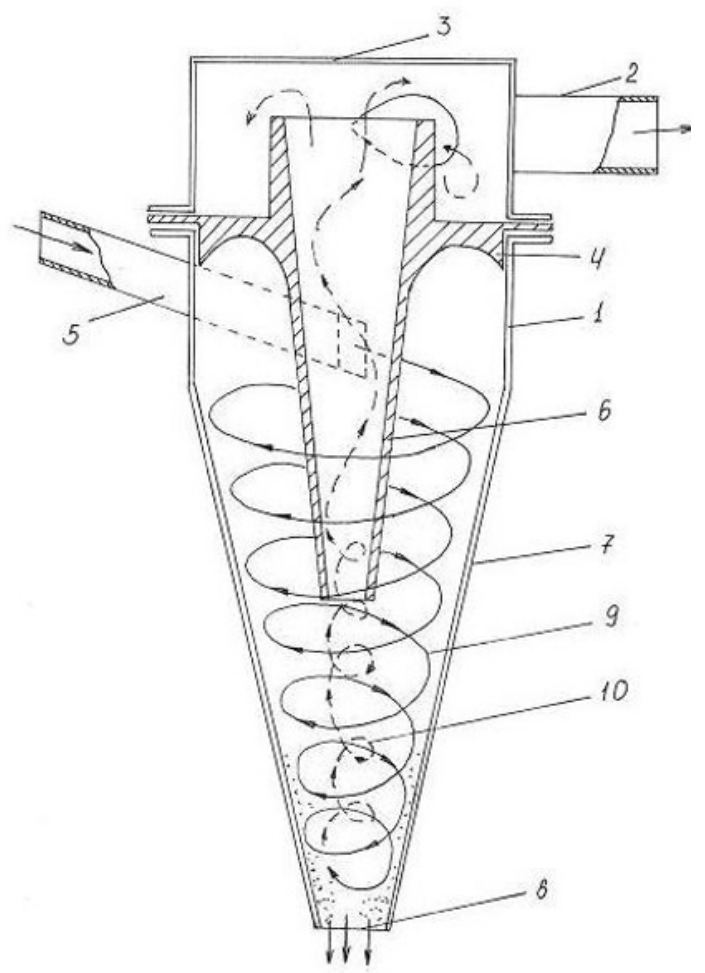

Fig. 1. Hydrocyclone diagram (explanations are in the text)

\section{Particle settling diameter determination}

\subsection{Theoretical investigations}

The separation process of emerged oils into fractions in a hydrocyclone can be represented as follows. The following forces act on a particle that is in a fluid flow in a hydrocyclone (fig. 2): the centrifugal force $\mathrm{Pz}$, throwing the particle to the periphery; the radial force $P_{r}$, arising the particle from the action of the radial fluid flow and directed to the apparatus axis; Coriolis force $\mathrm{P}_{k}$, which moves a particle in a circumferential direction relative to the flow; the resistance force of the environment $\mathrm{P}_{c}$, preventing the particle settling deposition; inertial force $\mathrm{P}_{u}$, which appears as a result of a particle settling speed change.

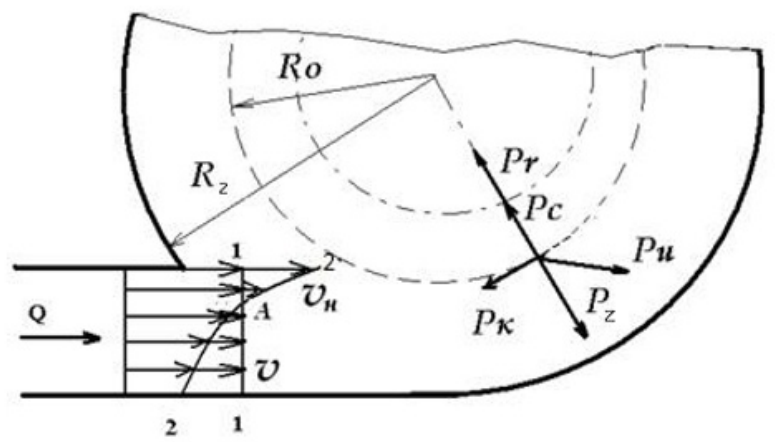

Fig. 2. Forces, acting on a particle in a spiral flow in a hydrocyclone

If the particle is small and carries along by the flow into rotational motion with an angular velocity $\omega$, then the main force acting on it is centrifugal one (Shestov, 1967)

$$
P_{z}=m \cdot \omega^{2} \cdot r=\frac{\pi \cdot \delta^{2} \cdot \rho \cdot \omega^{2} \cdot r}{6}=\frac{\pi \cdot \delta^{2} \cdot \rho \cdot v^{2}}{6 r}
$$

where $m-$ is the particle mass, $\mathrm{kg} ; \omega-$ is the angular velocity of rotation, $\mathrm{s}^{-1} ; r-$ is the particle rotation radius, $\mathrm{m} ; \delta-$ is the particle diameter, $\mathrm{m} ; \rho-$ is the particle density, $\mathrm{kg} / \mathrm{m}^{3} ; v-$ is the linear local flow velocity, $\mathrm{m} / \mathrm{s}$.

We can observe the Stock-Soviet (laminar) mode of particles sedimentation in the oil flow, ensuring their separation into phases by a spiral-like movement of the fluid flow in a hydrocyclone. If we take into account the centrifugal force $R_{z}$ action and the medium resistance force $P c$, then from expression (1) with consideration for the medium resistance force, we get:

$$
\frac{\pi \cdot \delta_{p}^{2} \cdot \rho_{p} \cdot v^{2}}{6 r}=\frac{24 \mu}{v_{s} \cdot \delta_{p} \cdot \rho_{l}} \cdot \frac{\pi \cdot \delta_{p}^{2}}{4} \cdot \frac{\rho_{l} \cdot v_{s}^{2}}{2}
$$

where $\rho_{l}$ is the liquid density, $\mathrm{kg} / \mathrm{m}^{3} ; v_{s}-$ is the separation rate (solid particles of insoluble impurities release from the oil), $\mathrm{m} / \mathrm{c} ; \mu$ is the kinematic viscosity of the oil being cleaned, $\mathrm{m}^{2} / \mathrm{s}$.

Then

$$
\frac{v^{2}}{r}=\frac{18 \cdot v_{s} \cdot \mu}{\delta_{p}^{2} \cdot \rho_{p}} .
$$

The inertial force $P_{u}$ is due to a change in the relative particle velocity in the medium flow when the particle velocity profile is rearranged at the entrance to the cylindrical part of the hydrocyclone. The fluid flow enters the cyclone with the initial velocity $v_{H}$ (the fluid velocity in the inlet nozzle of the cyclone); then the velocity profile changes along line $1-1$ (Fig. 2). The velocity profile $v_{H}$ (Fig. 2 . line $2-2$ ) is changed by a fluid transition into a curving duct of the inlet nozzle and its distribution over the inlet channel cross section corresponds to the law (Shestov, 1967) of variation the tangential velocity from radius.

$$
v r^{n}=\text { const }
$$

where $n$ is a freedom indicator

Then the fluid velocity distribution in the curvilinear channel (line 2-2 in Figure 2) will be determined by the dependence:

$$
v=\frac{v_{H}\left(R_{2}-R_{1}\right)}{r \ln \frac{R_{2}}{R_{1}}},
$$

where $R_{2}$ is the fluid flow outer radius, equal to the hydrocyclone radius $R_{2}=R_{z}, \mathrm{~m} ; R_{1}$ is the fluid flow internal radius in a hydrocyclone, $\mathrm{m}$

Substituting (5) in (3) we get:

$$
\frac{v_{i}\left(R_{2}-R_{1}\right)^{2}}{r^{3}\left(\ln \frac{R_{2}}{R_{1}}\right)^{2}}=\frac{18 \cdot v_{s} \cdot \mu}{\delta_{p}^{2} \cdot \rho_{p}} .
$$

Taking as a current radius $r$ its average value:

$$
r_{a}=\frac{R_{2}+R_{1}}{2}
$$


We get a generalized dependence characterizing the minimum diameter of the deposited particles:

$$
\delta_{p \text {.min }}=\sqrt{\frac{18 \cdot v_{s} \cdot \mu \cdot D}{2,5 \cdot v_{i}^{2} \cdot \rho_{p}}},
$$

where $D$ is cyclone diameter $D=2 R_{2}, \mathrm{~m}$

However, the use of the average value radius allows determining only the particles diameter locating at a given radius, and does not allow obtaining the real diameter of the deposited particle. To solve this problem, it is necesasry to use the radius value of zero vertical velocity. If we assume that there is no vertical effect on the particle in the cyclone flow, then each particle will be suspended at a certain distance from the center of rotation, that is, at a distance where the centrifugal force will be equal to the radial force $P_{z}=P_{r}$.

Heavier particles will be located closer to the hydrocyclone periphery, and light ones will be located at its center. Each group of particles in the oil flow in the inner conical hydrocyclone cavity will create a curvedlinear surface (Fig. 3) when they are moving.

Zero vertical velocity points (that is, the boundaries of the vertical velocity signs change) also create a curved surface. The intersection or coincidence of the vertical velocity plane with the plane of any group particles will give the value of the boundary grain, with the larger grain going to the drain and the smaller grain to the cleaned stream.

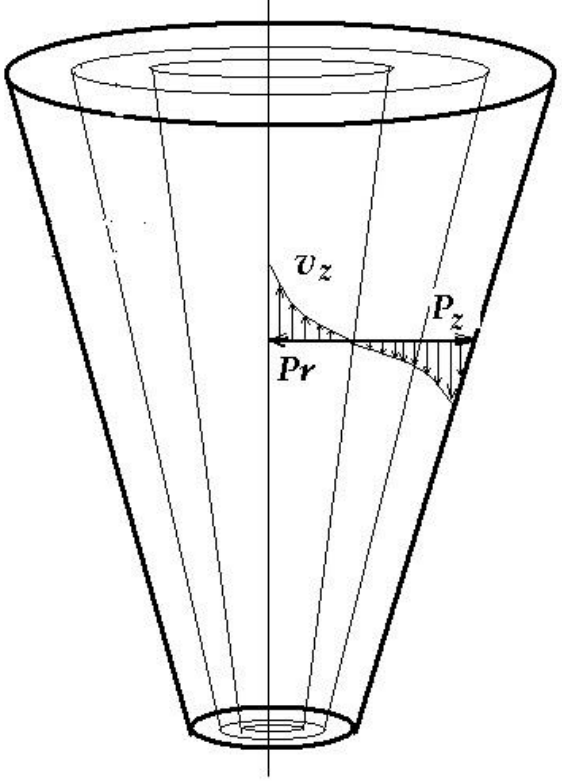

Fig. 3. Diagram of particle surfaces in a hydrocyclone flow

It is necessary to determine the axial velocity of a particle moving in a hydrocyclone flow to define the radius of the zero surface.

We use the formula to calculate the axial velocity [4]:

$$
\begin{aligned}
& v_{Z}=\frac{G}{4 \pi \cos \frac{\alpha_{1}}{2}} \int_{0}^{H+H 1} d h \int_{0}^{2 \pi} \frac{\left(R_{0}-h t g \frac{\alpha_{1}}{2}\right)}{\left[(h-z)^{2}+\left(R_{0}-h t g \frac{\alpha_{1}}{2}\right)^{2}\right.}, \\
& \frac{-\left(R_{0}-h t g \frac{\alpha_{1}}{2}\right) R \cos \beta}{\left.+R^{2}-2 R \cos \beta\left(R_{0}-h t g \frac{\alpha_{1}}{2}\right)\right]^{3 / 2}} d \beta
\end{aligned}
$$

where $G=$ const is the velocity circulation per unit of the toroid height

$$
G=2 \pi r v_{t}
$$

$R_{o}$ is the surface radius of zero axial velocities at the boundary of the cylindrical and conical parts of the hydrocyclone, $\mathrm{m}$

$$
R_{\hat{I}}=\frac{r_{o}}{r_{c}+r_{n}}\left(R_{G}+H \operatorname{tg} \frac{\alpha}{2}\right)
$$

where $R_{\Gamma}$ is the radius of the hydrocyclone, $\mathrm{m} ; r_{o}$ is the radius of the air column $\left(\mathrm{r}_{\mathrm{o}}=0,606 \mathrm{r}_{\mathrm{c}}\right), \mathrm{m} ; r_{c}$ is the radius of cleaned oil discharging, $\mathrm{m} ; r_{n}$ is the radius of the drain pipe, $\mathrm{m} ; \alpha_{l}$ is the taper angle of the hydrocyclone, deg.; $\beta$ is the angle of inclination or the offset angle of a point when moving in a vortex layer to a height (h-z), deg.; $h$ is the distance of the considered annular section from the beginning of the vortex layer (i.e, from the top lid of the hydrocyclone cylindrical part), $\mathrm{m} ; H$ is the height of the hydrocyclone conical part, $\mathrm{m} ; H_{1}$ is the height of the hydrocyclone cylindrical part, $\mathrm{m}$.

Tangential velocity of the particle

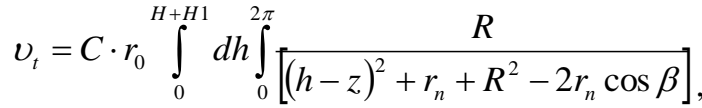

$$
\begin{aligned}
& \frac{-r_{n} \cos \beta}{\left(r_{n}^{2}+R^{2}-2 R_{0} \cos \beta\right)} d \beta
\end{aligned}
$$

where $C$ is a constant value characterizing the dependence of productivity on the hydrocyclone height ; $r_{H}$ is the outer diameter of the considered annular section, $\mathrm{m} ; R$ and $z$ are the coordinates of the point for which the velocity is determined, $\mathrm{m} ; v_{t}$ - tangential velocity of the particle, $\mathrm{m} / \mathrm{s}$; $d h$ is the height of the considered ring, $\mathrm{m}$;

$$
\operatorname{tg} \frac{\alpha_{1}}{2}=\frac{r_{o}}{r_{c}+r_{n}} \operatorname{tg} \frac{\alpha}{2}
$$

Axial velocity of a particle 


$$
\begin{aligned}
& v_{z}=\frac{2 \pi r C r_{0}}{4 \pi \cos \frac{\alpha_{1}}{2}} \int_{0}^{H+H 1} \int_{0}^{2 \pi}\left[(h-z)^{2}+r_{n}+R^{2}-2 r_{n} \cos \beta\right] \\
& \frac{-r_{0} \cos \beta}{\left(r_{0}^{2}+R^{2}-2 R_{0} \cos \beta\right)^{2}} \times \\
& \times \int_{0}^{H+H_{1}} d h \int_{0}^{2 \pi} \frac{\left[\left(R_{0}-h t g \frac{\alpha_{1}}{2}\right)^{2}\right.}{\left[(h-z)^{2}+\left(R_{0}-h t g \frac{\alpha_{1}}{2}\right)\right.} \\
& \left.\quad-\left(R_{0}-h t g \frac{\alpha_{1}}{2}\right) R \cos \beta\right] \\
& \left.+R^{2}-2 R \cos \beta\left(R_{0}-h t g \frac{\alpha_{1}}{2}\right)\right]^{\frac{3}{2}} d \beta d h
\end{aligned}
$$

In order to establish a criterial form communication in the form of a regression model of the zero surface radius dependence $R_{o}$ on the hydrocyclone parameters, we compose a matrix, taking as predictors: the height of the hydrocyclone working part $H+H_{l}$, the particle coordinate according to the hydrocyclone radius $R$, the particle coordinate according to the hydrocyclone height $H+H_{l}$, distance to the considered section $h$ of the hydrocyclone conical part, diameter of the section $d$, axial velocity $v_{z}$ (Table 1$)$.

Table 1. Matrix for the establishment of communication in the criterial form

\begin{tabular}{|c|c|c|c|c|c|c|}
\hline $\boldsymbol{R}_{\boldsymbol{o}}$ & $\boldsymbol{H}+\boldsymbol{H}_{\boldsymbol{1}}$ & $\boldsymbol{R}$ & $\boldsymbol{z}$ & $\boldsymbol{h}$ & $\boldsymbol{d}$ & $\boldsymbol{v}_{z}$ \\
\hline-1 & -1 & -1 & -1 & -1 & -1 & -1 \\
\hline-1 & -1 & -1 & -1 & -1 & -1 & 0 \\
\hline-1 & -1 & -1 & -1 & -1 & 0 & 1 \\
\hline-1 & -1 & -1 & -1 & 0 & 1 & 1 \\
\hline-1 & -1 & -1 & 0 & 1 & 1 & 1 \\
\hline-1 & -1 & 0 & 1 & 1 & 1 & 1 \\
\hline
\end{tabular}

The regression equation will be

$$
R_{o}=a_{o}+a_{1} X_{1}+a_{2} X_{2}+\ldots+a_{n} X_{n}=0
$$

where $a_{o}, a_{1}, \ldots a_{n}$ are regression coefficients with selected parameters of a hydrocyclone; $X_{1}, X_{2}, \ldots X_{n}$ are the parameters of the hydrocyclone.

Having preliminary information about the influence of the hydrocyclone listed parameters on the radius of the surface of zero axial velocity, we present the functional dependence in general form

$$
R_{o}=f(\alpha, R, h, z, d) \text {. }
$$

To calculate the coefficients, we determine the change in the axial velocity and the coordinates of a particle moving in a vortex flow of a hydrocyclone at three points of the section along the height and ten points along the radius of the hydrocyclone $[5,6,7]$. In this case, the particle coordinate $z$ will take certain numerical values depending on the diameter of the section, and accordingly, on its position along the height of the hydrocyclone. Given the condition that the $z$ coordinate is a numerical variable value, we will get:

$$
r_{o}, R_{o}, \alpha, \beta, r, h=\text { const; }
$$

that is, only the coordinate of the point being examined will change $R$. After calculating the position of the point, we will obtain a series of numerical values of the main parameters.

\section{Research results}

A quadratic equation of the axial velocity of the particle in the flow is obtained after determining the coordinates of changing the position of a particle in a hydrocyclone flow and calculating the regression coefficients. After substituting into it the boundary values of the predictors defined for these specific conditions $(h=0,01-0,18 \mathrm{~m}$, $R_{0}=0,02 \mathrm{~m}, r_{o}=0,017 \mathrm{~m}, \alpha=5^{\circ} 2^{\prime}, H+H_{o}=0,302 \mathrm{~m}, \beta=$ $\left.100-120^{\circ}, R=0,018-0,046 \mathrm{~m}\right)$, and transformations, a theoretical dependence of the axial velocity of a particle in a flow, on the design and technological parameters of a hydrocyclone depending on the required values of the quality of oil cleaning is obtained.

$$
\begin{aligned}
& v_{z}=Y=(-244,524-30)-19769,382 r- \\
& -138,852 z+31610,804 C+1373,869 r^{2}+ \\
& +227914,938 r^{2}-17399,748 z^{2}+ \\
& +158265,452 C^{2}-88475,75 R^{2}
\end{aligned}
$$

For the calculations of the boundary layer radius (zero surface), the dependence of the hydrocyclone parameters is obtained: radius $R$, height (as a function of performance) $C$ on the particle coordinate in the cleaning flow $z$ :

$$
R=0,0385-0,833 r-0,0072 z-0,347 C,
$$

Substitute zero surface radius equation (17) of the axial velocity in expression (6), replacing the value of the diameter of the hydrocyclone $D$ by the radius of the zero surface $2 R_{o}$. Then we will get the size of the particles separated at a certain radius of the cyclone:

$$
\delta_{p, \min }=\sqrt{\frac{36 v_{c} \mu R_{o}}{2,5 v_{n}^{2} \rho_{p}}}
$$

The obtained dependence clearly shows the relationship of each quantity in the radicand with the size of the particles separated in the spiral flow of the hydrocyclone. At the same time, the size of separated particles will increase with the increase in the radius of the zero surface. Therefore, reduced diameters hydrocyclones should be used to trap fine particles [5, $6]$. Increasing of the initial flow velocity of the cleaned oil $v_{u}$ (in the cyclone inlet) also contributes to the separation of smaller particles.

The using of expression (18) to determine the minimum size of a detachable particle for practical purposes is not always possible, since it contains an unknown quantity - the separation velocity $v_{\mathrm{c}}$.

To determine the separation velocity, we assume that the particle entering the cyclone near the inlet nozzle (radius $R_{1}$ ) must settle on the wall of the cyclone during its passing through the cylindrical-conical part of the cyclone.

$$
\tau=\frac{R_{2}-R_{1}}{v_{c}}=\frac{V_{h}}{Q_{h}}=\pi H \frac{R_{2}-R_{1}}{Q_{h}},
$$

where $V_{h}$ is the volume of the hydrocyclone cylindricalconical part, $\mathrm{m}^{3} ; Q_{h}$ is the hydrocyclone productivity, 
$\mathrm{m}^{3} / \mathrm{h} ; H$ is the height of the hydrocyclone cylindricalconical part, $\mathrm{m}$.

Whereas the separation of particles occurs at radius $R_{o}$, replacing $R_{2}-R_{l}$ with $R_{o}$, we get

$$
\tau=\pi H \frac{R_{\hat{\imath}}}{Q_{h}}
$$

In this case, the particle settling rate

$$
v_{\tilde{n}}=\frac{Q_{h}}{\pi H R_{o}}
$$

Three quantities are essential for separation of a particle in a hydrocyclone: the separation rate $v_{\mathrm{c}}$, the thickness of the deposition layer and the residence time of the particle in the hydrocyclone $\tau$.

A parameter can be made up of these three quantities - a separation criterion that determines the amount of particles to be separated from the purified oil stream.

$$
K_{c}=\frac{v_{c} \tau}{\lambda}
$$

where $\lambda$ is the thickness of the deposition layer, $\mathrm{m}$.

Since the thickness of the deposition layer is determined by the boundary values of the radii $R_{z}-R_{o}$, then

$$
\lambda=R_{z}-R_{o}
$$

Since the quality of cleaning determines the amount of deposited impurities, we replace the volume of supplied oil with the amount of impurities in the cleaned oil. Performing the replacement and substituting the value of $v_{c}$ in the formula (22), we get

$$
K_{c}=\frac{G_{h} \tau R_{z}}{\pi H},
$$

where $G_{h}$ is the amount of impurities in the cleaned oil, $\mathrm{kg}$.

In this case, the quality of hydrocyclone cleaning can be defined as the particles entrainment through a drain hole, expressed as a percentage of the particles total number entering the hydrocyclone with the cleaned oil:

$$
\phi=\frac{G_{h}-K_{c}}{G_{h}} .
$$

Thus, the quality of oil purification will be determined by the geometrical parameters of the hydrocyclone and the residence time of the particle in the hydrocyclone, depending on the separation mode.

The comparative laboratory studies of the efficiency of purification of spent mineral oil from insoluble impurities using experimental and controlling hydrocyclones were carried out to confirm the obtained theoretical calculations. A hydrocyclone made according to the calculating method of its geometrical parameters recommended by most researchers was used as a controlling [8-10].

In the process of research, it was found that an experimental cylindro-conical hydrocyclone, with geometrical parameters set in accordance with the theoretical calculations, provides the best degree of purification $(88.4 \%)$ with a process productivity of $3 . .5$ $\mathrm{t} / \mathrm{h}$, input flow pressure $P=0.04 \mathrm{MPa}$ and the diaphragm seal immersion depth of a hydrocyclone $H=136.8 \mathrm{~mm}$, contrary to $21.6 \%$ for a typical hydrocyclone.

\section{Conclusion}

The purification degree of oil from insoluble impurities will be determined by the geometrical parameters of the hydrocyclone: radius $R_{z}$, the height of the cylindricalconical part $H$ and the time the particle stays in the hydrocyclone, depending on the separation mode. The results of the comparative laboratory studies show that the calculations of the structural and technological parameters of the hydrocyclone according to the proposed method provides a higher degree of purification of spent mineral oils from insoluble impurities.

\section{References}

1. A.A. Glushchenko, D.E. Molochnikov, S.A. Yakovlev, I.N. Gayaziev, To the issue of purification of spent oils from insoluble impurities in a hydrocyclone Vestnik of Kazan state agrarian Univer. 3(50), 81-84 (2018)

2. K.U. Safarov, V.M. Kholmanov, M.M. Zamaldinov, Restoration of motor oils by the step method Vestnik of Ulyanovsk state agricultural Academy 3, 84-87 (2000)

3. S.A. Kolokoltsev, M.M. Zamaldinov, Changing the quality of engine oil during operation of an internal combustion engine Sci. in Central Russia. Tambov 4S, 38-40 (2013)

4. E.S. Zamaldinov, K.U. Zykin, Filter for cleaning used engine oil Patent for utility model RUS no. 107704 (26 April 2011)

5. M.M. Zamaldinov, Removal of mechanical impurities and water from used engine oil by gravity sedimentation method in Interuniversity collection of sci. papers of the XVI Region Sci. and Pract. Conf. of Univer. of the Volga and Ural Regions "Improving the use of automotive and agricultural machinery”, ed. V.D. Korotnev, 170-173 (Penza, State Agricultural Academy, 2005)

6. K.U. Safarov, M.M. Zamaldinov, Problems of the petroleum products secondary use at the present stage in Mat. of the Russian national Sci. and Pract. Conf. "Modern Development of the AIC: Regional Experience, Problems, Prospects”, eds. V.G. Artemyev, A.Kh. Kulikova, V.M. Kholmanov, 260-261 (Ulyanovsk, State Agricultural Academy, 2005)

7. V.M. Kholmanov, M.M. Zamaldinov, Determination of the hydrocyclone optimal operating mode in Mat. of the Russian national Sci. and Pract. Conf. "Modern Development of the AIC: Regional Experience, Problems, Prospects”, eds. V.G. Artemyev, A.Kh. Kulikova, V.M. Kholmanov, 261-263 (Ulyanovsk: State Agricultural Academy, 2005)

8. K.U. Safarov, V.M. Kholmanov, M.M. Zamaldinov, The study of improving the motor oils quality Bull. 
of the Ulyanovsk State Agricultural Acad. 3, 65-67 (State Agricultural Academy, Ulyanovsk, 2000)

9. A.N. Zazulya, A.A. Glushchenko, D.E. Molochnikov, M.A. Karpenko, G.V. Karpenko, Theoretical substantiation of the cylindrical-conical hydrocyclone geometric parameters influence on the degree of waste lubricating purification oils from insoluble impurities Sci. in Central Russia 2(38), 116-123 (2019)
10. M.M. Zamaldinov, K.U. Safarov, S.A. Kolokoltsev, The regeneration of spent mineral motor oils by centrifugation in Collec. of articles of the Russian national sci.-pract. Conf. "Operation of automotive machinery: experience, problems, innovations, prospects" 39-42 (State Agricultural Academy, Ulyanovsk, 2013) 